\title{
A Novel Decentralized Relative Navigation Algorithm for Spacecraft Formation Flying
}

\author{
Xiaogang Wang, Qin Wutao † Bai Yuliang: Naigang Cui \$ \\ Harbin Institute of Technology, Harbin, 150001, China
}

\begin{abstract}
This paper presents a novel decentralized relative navigation algorithm. The relative motion equations are derived in the Earth-Centered-Inertial frame. The relative measurements contain not only the line of sight and range between the deputy and the chief, but also the ranges among different deputies. This helps to improve the redundancy and accuracy of the relative navigation for spacecraft formation flying. In decentralized estimation algorithm, it is necessary to transmit the global states in the formation to linearize the ranges among the deputies. A sigma-point method is used to account for the uncertainty in the estimated states of other spacecraft. The relative measurements are coupled with the relative motion equation in a novel decentralized filter to determine the relative position and velocity. Simulation shows that the proposed novel decentralized estimation algorithm provides better performance than the traditional iteration algorithm.
\end{abstract}

Keywords: Relative navigation; Formation flying; Decentralized estimation algorithm; sigma-point filter; Relative motion

\section{Introduction}

Spacecraft formation flying (SFF) is an important technology for future missions, such as rendezvous and docking, long baseline interferometry, stereographic imaging and synthetic apertures. Many researches have been done with respect to spacecraft formation flying, such as relative dynamics, motion control and navigation $[1,2,3]$. Relative navigation $[4,5,6]$ would be the topic in this paper.

\footnotetext{
*Associate Professor, Department of Aerospace Engineering, 92 XiDaZhi Street

${ }^{\dagger}$ PHD Candidate, Department of Aerospace Engineering, 92 XiDaZhi Street.

¥Assistant Professor, Department of Aerospace Engineering, 92 XiDaZhi Street.

$\S$ Professor, Department of Aerospace Engineering, 92 XiDaZhi Street.

'Corresponding Author Email Address: wangxiaogang@hit.edu.cn
} 
In the past, researches on relative navigation of SFF have been focused on methods using GPS (Global Positioning System) [7, 8]. However, this method is not suitable for high earth orbit (HEO) and deep-space formation flying because the GPS signal is not available in these cases. A GPS-like technology has been proposed for deep-space mission, however, this technique requires extensive hardware and is subject to some similar error sources to GPS [9]. Recently, vision-based navigation (VISNAV) system has been proposed by using an optical sensor with specific light sources [10, 11]. The VISNAV provides mutiple line-of-sight between the deputies and chief which could be coupled with gyro measurement and dynamic models in an extended kalman filter to determine relative attitude, position and gyro biases. However, the VISNAV is not suitable for long distance application, in which case multiple line of sight will converge to single line of sight.

In this paper, the relative measurements contain both the line-of-sight and range between the deputy and the chief and the ranges between all deputies. By accquiring the ranges among the deputies, the relative navigation system improves both the redundancy and the accuracy. Moreover, the proposed relative system can also be applied to missions where GPS signal is not available, for example, high earth orbit and deep-space missions. There are three basic estimation architectures including centralized, decentralized and hierarchic. Generally, the centralized architecture could get the most accurate estimation result, but is subject to high computational burden in the chief and frequent communication demands among formation members. Therefore, it is desirable to select decentralized architecture. Because the computation of the ranges between the deputies requires the relative states of spacecraft in formation, the key issue to use decentralized estimation algorithm is to account for the uncertainty in the relative states of spacecrafts. Some papers presented several decentralized algorithms to compensate the uncertainty, including the Iterative Cascade Extended Kalman Filter, Schmidt Kalman Fiter, Bump-up-R algorithm $[12,13,14]$ and other decentralized filter $[15,16,17]$. In this paper, we propose to incorporate sigma-point method to account for the uncertainty.

The organization of this paper is as follows. Section 2 presents the system equations including relative motion equations and measurement equations. In Section 3, a novel decentralized estimation algorithm is developed and the decentralized filter is designed. To account for uncertainty, sigma-point method is employed. Simulations are conducted and the results are presented in Section 4 to verify the effectiveness. Finally, Section 5 concludes this paper with some conclusions. 


\section{System Equations}

In this section, we will derive the relative motion equations for formation flying and the measurement equations for both vision and range sensors.

\subsection{Relative Motion Equations}

The spacecraft are identified as the "chief" and the "deputies". The spacecraft about which all other space- craft are orbiting is referred to as the chief. The remaining spacecraft are referred to as the deputies. The inertial equations of the chief's motion could be given by the standard Newtonian relationship

$$
\ddot{\mathbf{r}}_{c}=-\mu \frac{\mathbf{r}_{c}}{r_{c}^{3}}
$$

Where $\mu$ is the Earth gravitational constant, $\mathbf{r}_{c}=\left[\begin{array}{lll}x_{c} & y_{c} & z_{c}\end{array}\right]^{T}$ is the position vector in ECI frame, the subscript $c$ denotes the chief spacecraft, and

$$
r_{c}=\left\|\mathbf{r}_{\mathbf{c}}\right\|=\sqrt{x_{c}^{2}+y_{c}^{2}+z_{c}^{2}}
$$

Similarly, the deputy inertial equations of motion are

$$
\ddot{\mathbf{r}}_{d}=-\mu \frac{\mathbf{r}_{d}}{r_{d}^{3}}+\mathbf{v}+\mathbf{f}_{c}
$$

Where $\mathbf{r}_{d}=\left[\begin{array}{lll}x_{d} & y_{d} & z_{d}\end{array}\right]^{T}$, the subscript $d$ denotes the deputy spacecraft, the vector $\mathbf{v}$ is the perturbing force, $\mathbf{f}_{c}$ is the control force, and

$$
r_{d}=\left\|\mathbf{r}_{d}\right\|=\sqrt{x_{d}^{2}+y_{d}^{2}+z_{d}^{2}}
$$

The relative position vector $\mathbf{r}_{c d}=\left[\begin{array}{lll}x_{c d} & y_{c d} & z_{c d}\end{array}\right]^{T}$ is defined as

$$
\mathbf{r}_{c d}=\mathbf{r}_{d}-\mathbf{r}_{c}
$$

So,

$$
\mathbf{r}_{d}=\mathbf{r}_{c d}+\mathbf{r}_{c}
$$


Combining Eqs. $(1,3,4)$ and neglecting the control forces $\mathbf{f}_{c}$, it yields

$$
\begin{aligned}
\ddot{\mathbf{r}}_{c d} & =\ddot{\mathbf{r}}_{d}-\ddot{\mathbf{r}}_{c} \\
& =\mu \frac{\mathbf{r}_{c}}{r_{c}^{3}}-\mu \frac{\mathbf{r}_{d}}{r_{d}^{3}}+\mathbf{v}
\end{aligned}
$$

Substituting Eq. (6) into Eq. (7) gives

$$
\ddot{\mathbf{r}}_{c d}=\mu \frac{\mathbf{r}_{c}}{r_{c}^{3}}-\mu \frac{\mathbf{r}_{c d}+\mathbf{r}_{c}}{\left\|\mathbf{r}_{c d}+\mathbf{r}_{c}\right\|^{3}}+\mathbf{v}
$$

Where

$$
\left\|\mathbf{r}_{c d}+\mathbf{r}_{c}\right\|^{3}={\sqrt{\left(x_{c d}+x_{c}\right)^{2}+\left(y_{c d}+y_{c}\right)^{2}+\left(z_{c d}+z_{c}\right)^{2}}}^{3}
$$

Eqs. (8-9) are the relative motion equations used in this study, and the perfect orbit knowledge for the chief spacecraft is assumed.

\subsection{Measurement Equations}

Vision sensor and range sensor are located on the deputy spacecraft. The vision sensor provides the line-of-sight between the deputy and the chief. The range sensors provide the range information from a deputy to the chief and to other deputies.

\subsubsection{Vision Sensor}

The vision sensor provides the line-of-sight vector from the deputy to the chief in the deputy's body frame. The attitude of the deputy is assumed to be known exactly. Therefore, the elevation and azimuth angle can be expressed in the ECI as shown in Fig. 1. The line-of-sight vector $\mathbf{l}_{d c}$ can be expressed in the $O \mathbf{X}_{I} \mathbf{Y}_{I} \mathbf{Z}_{I}$ frame as

$$
\mathbf{I}_{d c}=\cos \alpha \cos \beta \mathbf{I}_{x}+\cos \alpha \sin \beta \mathbf{I}_{y}+\sin \alpha \mathbf{I}_{z}
$$

where $\alpha$ is the elevation angle, $\beta$ is the azimuth angle. The line-of-sight vector $\mathbf{1}_{d c}$ and relative position vector $\mathbf{r}_{c d}$ are in an opposite direction, so 


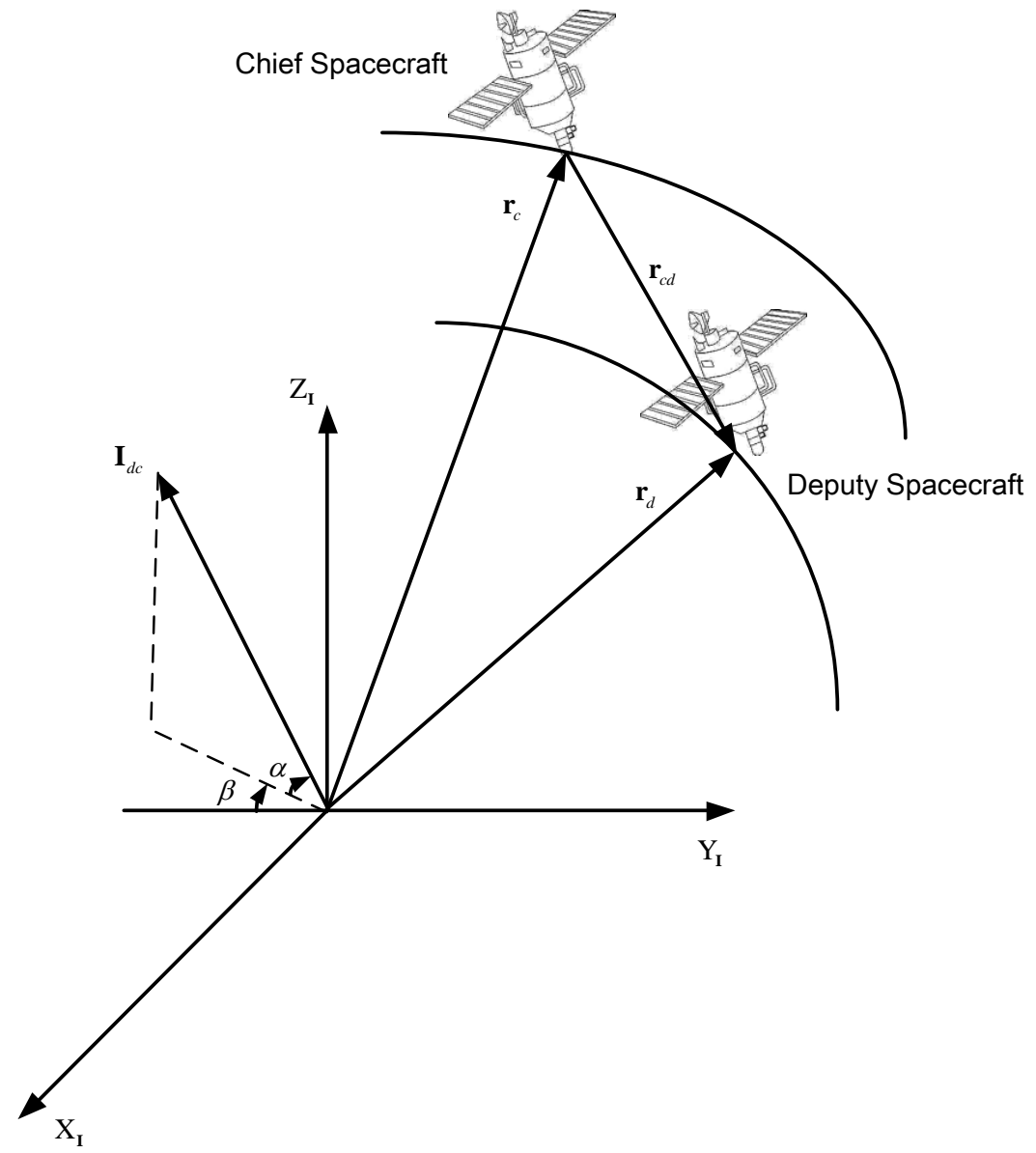

Fig. 1 Line of Sight Measurement

$$
\begin{gathered}
\alpha=-\arcsin \frac{z_{c d}}{r_{c d}} \\
\beta=\arctan \frac{y_{c d}}{x_{c d}}
\end{gathered}
$$

Where

$$
r_{c d}=\sqrt{x_{c d}^{2}+y_{c d}^{2}+z_{c d}^{2}}
$$

\subsubsection{Range Sensor}

All deputies are equipped with range sensors to measure the ranges between a deputy and other spacecraft (chief and other deputies). 


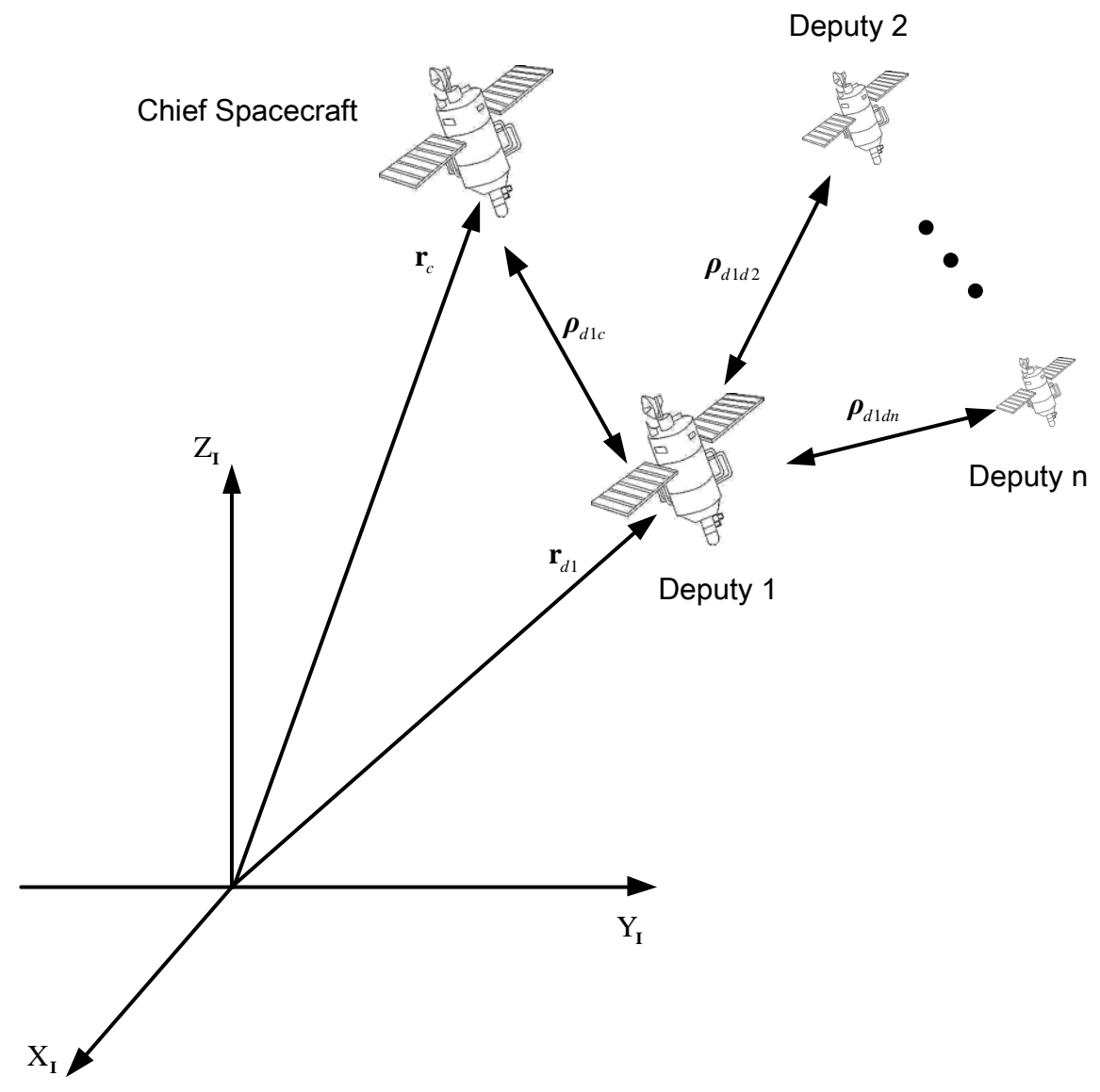

Fig. 2 Range Measurement of Deputy 1

For example the deputy 1 shown in Fig. 2, the range $\rho_{d 1 c}$ between it and the chief is

$$
\rho_{d 1 c}=\sqrt{x_{c d 1}^{2}+y_{c d 1}^{2}+z_{c d 1}^{2}}
$$

Where $\mathbf{r}_{c d 1}$ is the relative position vector between the deputy 1 and the chief, and $x_{c d 1}, y_{c d 1}, z_{c d 1}$ are the components of $\mathbf{r}_{c d 1}$.

The range $\rho_{d 1 d i}$ between deputy 1 and the $i$-th deputy is

$$
\rho_{d 1 d i}=\sqrt{\left(x_{c d 1}-x_{c d i}\right)^{2}+\left(y_{c d 1}-y_{c d i}\right)^{2}+\left(z_{c d 1}-z_{c d i}\right)^{2}}
$$

Where $\mathbf{r}_{c d i},(i=2, \cdots, n)$ is the relative position vector between the deputy 1 and the $i$-th deputy, and $x_{c d i}, y_{c d i}, z_{c d i}$ are the components of $\mathbf{r}_{c d i}$. Therefore, there will be $n$ ranges measured by the range sensor on the deputy 1 . 


\section{Decentralized Filter Design}

In this study, we define the states of each spacecraft as the local states. The global states are defined as the summation of all local states. In decentralized estimation algorithm, every spacecraft estimates its local states, and contributes equally to the estimation of the global states. Since all spacecraft require the states of other spacecraft to linearize the range between them, it is necessary to transmit the global states among the formation. Park presented several decentralized filters, and pointed out that the key issue during estimating the local state is to account for the uncertainty in the states of other spacecraft [13].The filters developed in [13]include the Iterative Cascade Extended Kalman Filter, Schmidt Kalman Filter and Bump-up-R algorithm. In this paper, a novel decentralized estimation algorithm is proposed where the sigma-point[18] method is employed to account for the uncertainty in other spacecraft states.

\subsection{Decentralized Estimation Procedure}

The decentralized estimation process as shown in Fig. 3 can be summarized as follows:

- Step 1: Every spacecraft carries out the time update. The global states and covariance matrices are built up base on the local states.

- Step 2: The deputy 1 runs its local filter based on its measurements, the global states and covariance matrices.

- Updating its local information in the global states and the covariance matrices.

- Sending the global states and covariance matrices to the next spacecraft.

- Step 3: Repeat Step 2 for all remaining spacecraft.

- Step 4: Step 2 and 3 will be iterated, if necessary. 


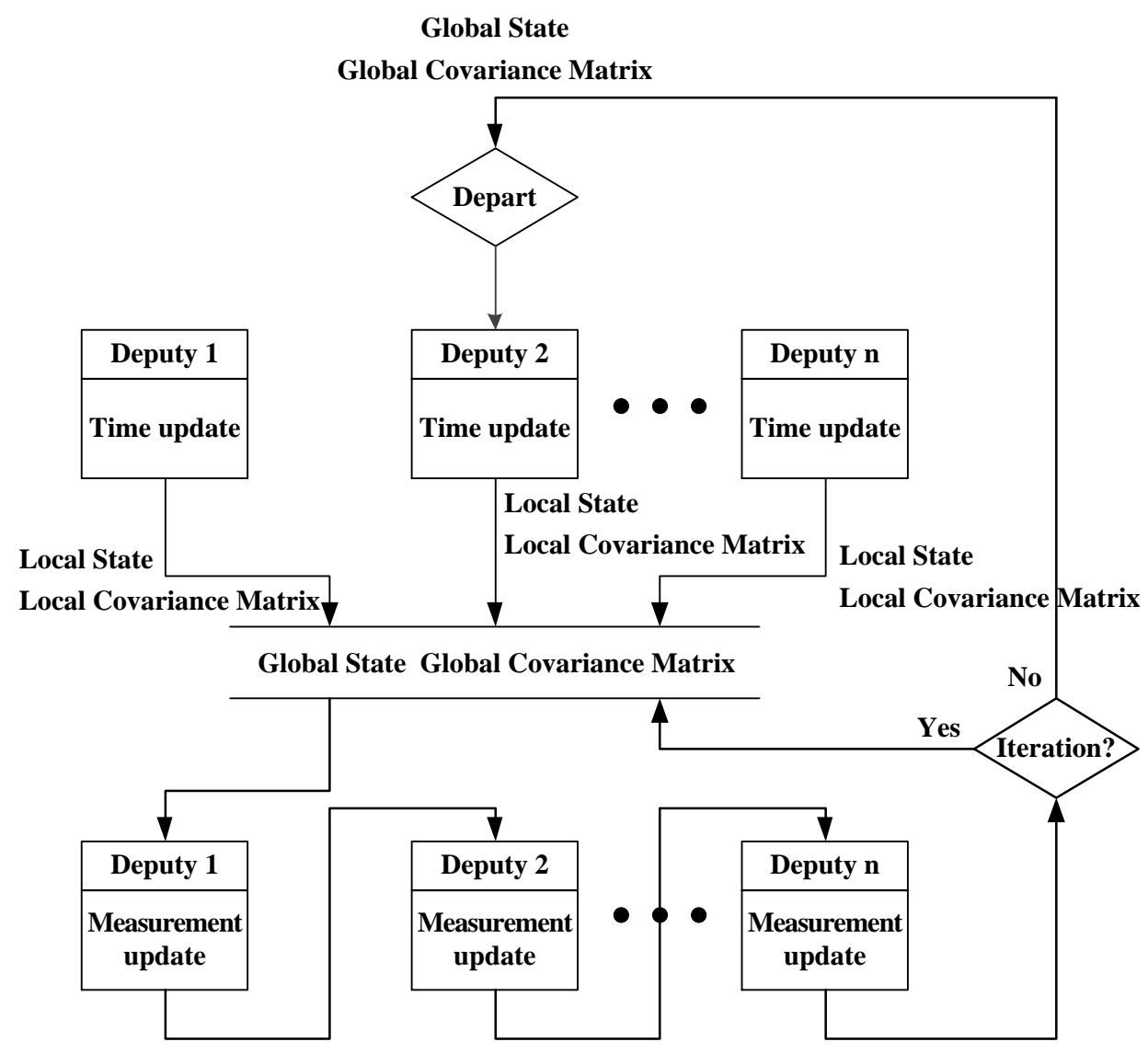

Fig. 3 Decentralized Estimation Procedure

\subsection{Time update}

Taking the deputy 1 for example, the time update equations are given as follows.

First, the relative position/velocity vector of deputy 1 relative to the chief is

$$
\mathbf{X}_{c d 1}=\left[\begin{array}{llllll}
x_{c d 1} & y_{c d 1} & z_{c d 1} & \dot{x}_{c d 1} & \dot{y}_{c d 1} & \dot{z}_{c d 1}
\end{array}\right]^{T}
$$

According to the Eq. (8), the nonlinear state model is

$$
\dot{\mathbf{X}}_{c d 1}=\mathbf{f}\left(\mathbf{X}_{c d 1}\right)=\left[\begin{array}{c}
\dot{x}_{c d 1} \\
\dot{y}_{c d 1} \\
\dot{z}_{c d 1} \\
\mu \frac{x_{c}}{r_{c}^{3}}-\mu \frac{x_{c d 1}+x_{c}}{\left\|\mathbf{r}_{c d 1}+\mathbf{r}_{c}\right\|^{3}}+v_{1} \\
\mu \frac{y_{c}}{r_{c}^{3}}-\mu \frac{y_{c d 1}+y_{c}}{\left\|\mathbf{r}_{c d 1}+\mathbf{r}_{c}\right\|^{3}}+v_{2} \\
\mu \frac{z_{c}}{r_{c}^{3}}-\mu \frac{z_{c d 1}+z_{c}}{\left\|\mathbf{r}_{c d 1}+\mathbf{r}_{c}\right\|^{3}}+v_{3}
\end{array}\right]
$$


So, the error-state vector becomes

$$
\Delta \mathbf{X}_{c d 1}=\left[\begin{array}{llllll}
\Delta x_{c d 1} & \Delta y_{c d 1} & \Delta z_{c d 1} & \Delta \dot{x}_{c d 1} & \Delta \dot{y}_{c d 1} & \Delta \dot{z}_{c d 1}
\end{array}\right]^{T}
$$

The matrix used in the covariance propagation is

$$
\mathbf{F}=\left[\begin{array}{cc}
\mathbf{0}_{3 \times 3} & \mathbf{I}_{3 \times 3} \\
\frac{\partial \mathbf{f}\left(\mathbf{X}_{c d 1}\right)}{\partial \mathbf{r}_{c d 1}} & \mathbf{0}_{3 \times 3}
\end{array}\right]
$$

Therefore, the propagation of relative state is the integral of Eq. (17), the propagation of covariance matrix $\mathbf{P}_{k}^{+}$could be given by

$$
\mathbf{P}_{k+1}^{-}=\boldsymbol{\Phi}_{k} \mathbf{P}_{k}^{+} \boldsymbol{\Phi}_{k}^{T}+\mathbf{Q}_{k}
$$

The state transition matrix is given by

$$
\boldsymbol{\Phi}_{k}=\mathbf{I}+\mathbf{F}_{k} \Delta t
$$

\subsection{Measurement Update}

The measurements of the deputy 1 include two parts:

(a) line of sight and range between deputy 1 and the chief.

(b) ranges between deputy 1 and other deputies.

For measurement (a), we have

$$
\begin{gathered}
\alpha_{k+1}^{-}=-\arcsin \frac{z_{c d 1, k+1}^{-}}{r_{c d 1, k+1}^{-}} \\
\beta_{k+1}^{-}=\arctan \frac{y_{c d 1, k+1}^{-}}{x_{c d 1, k+1}^{-}} \\
\rho_{d 1 c, k+1}^{-}=\sqrt{\left(x_{c d 1, k+1}^{-}\right)^{2}+\left(y_{c d 1, k+1}^{-}\right)^{2}+\left(z_{c d 1, k+1}^{-}\right)^{2}}
\end{gathered}
$$

For measurement (b), to account for the uncertainty in $\rho_{d 1 c, k+1}^{-}$due to $\hat{\mathbf{r}}_{c d 2, k+1}$, the augment state is introduced as

$$
\hat{\mathbf{X}}_{k+1}^{a}=\left[\begin{array}{c}
\hat{\mathbf{r}}_{c d 2, k+1} \\
0
\end{array}\right]
$$


and the covariance matrix is given by

$$
\mathbf{P}_{k+1}^{a}=\left[\begin{array}{cc}
\mathbf{P}_{r c d 2, k+1} & 0 \\
\mathbf{0}_{1 \times 3} & R_{n}
\end{array}\right]
$$

where the $\mathbf{P}_{r c d 2, k+1}$ is the covariance of $\hat{\mathbf{r}}_{c d 2, k+1}$.

According to the augmented state $\hat{\mathbf{X}}_{k+1}^{a}$, the Sigma-Point $\chi_{k+1}^{a}$ could be calculated as [19]

$$
\boldsymbol{\chi}_{k+1}^{a}=\left[\begin{array}{lll}
\hat{\mathbf{X}}_{k+1}^{a} & \hat{\mathbf{X}}_{k+1}^{a}+\gamma \sqrt{\mathbf{P}_{k+1}^{a}} & \hat{\mathbf{X}}_{k+1}^{a}-\gamma \sqrt{\mathbf{P}_{k+1}^{a}}
\end{array}\right]
$$

The measurement corresponds to $i$-th sigma-point is

$$
\mathcal{Y}_{i, k+1}=\left\|\mathbf{r}_{c d 1, k+1}^{-}-\chi_{i, k+1}^{c d 2}\right\|+\chi_{i, k+1}^{n}
$$

Therefore, the range $\rho_{d 1 d 2, k+1}^{-}$can be estimated by

$$
\rho_{d 1 d 2, k+1}^{-}=\sum_{i=0}^{2 L} \omega_{i}^{m} y_{i, k+1}
$$

Hence, the noise $R_{u, d 1 d 2}$ which accounts for the uncertainty of the deputy 2 is

$$
R_{u, d 1 d 2}=\sum_{i=0}^{2 L} \omega_{i}^{c}\left(y_{i, k+1}-\rho_{d 1 d 2, k+1}^{-}\right)^{2}
$$

Where $\gamma$ is a composite scaling parameter and could be given by

$$
\begin{gathered}
\gamma=\sqrt{L+\lambda} \\
\lambda=\delta^{2}(\lambda+\kappa)-L
\end{gathered}
$$

where $L=4$ is the dimension of the augment state, the default choice of $\kappa$ is 0 , the parameter $\delta$ controls the "size" of the Sigma-Point distribution, and could be chosen between 0 and 1 .

The weight $\omega^{m}$ and $\omega^{c}$ are

$$
\begin{gathered}
\omega_{0}^{m}=\frac{\lambda}{\lambda+L} \\
\omega_{0}^{c}=\frac{\lambda}{\lambda+L}+\left(1-\delta^{2}+\xi\right) \\
\omega_{0}^{c}=\omega_{0}^{m}=\frac{1}{2(\lambda+L)} \quad i=1, \ldots, 2 L
\end{gathered}
$$


Where $\xi$ is a non-negative weighting term which can be used to incorporate knowledge of the higher order moments of the distribution. For a Gaussian prior, the optional choice is $\xi=2$.

The sensitivity matrix $\mathbf{H}_{k+1}$ is

$$
\mathbf{H}_{k+1}=\left[\begin{array}{cc}
\frac{\partial \alpha}{\partial \mathbf{r}_{c d 1}} & \mathbf{0}_{1 \times 3} \\
\frac{\partial \beta}{\partial \mathbf{r}_{c d 1}} & \mathbf{0}_{1 \times 3} \\
\frac{\partial \rho_{d 1 c}}{\partial \mathbf{r}_{c d 1}} & \mathbf{0}_{1 \times 3} \\
\frac{\partial \rho_{d 1 d 2}}{\partial \mathbf{r}_{c d 1}} & \mathbf{0}_{1 \times 3} \\
\cdots & \cdots \\
\frac{\partial \rho_{d 1 d n}}{\partial \mathbf{r}_{c d 1}} & \mathbf{0}_{1 \times 3}
\end{array}\right]
$$

The measurement noise matrix is

$$
\mathbf{R}_{k+1}=\left[\begin{array}{ccccccc}
R_{\alpha} & 0 & 0 & 0 & 0 & \cdots & 0 \\
0 & R_{\beta} & 0 & 0 & 0 & \cdots & 0 \\
0 & 0 & R_{n} & 0 & 0 & \cdots & 0 \\
0 & 0 & 0 & R_{u, d 1 d 2} & 0 & \cdots & 0 \\
0 & 0 & 0 & 0 & R_{u, d 1 d 3} & \cdots & 0 \\
\cdots & \cdots & \cdots & \ldots & \cdots & \cdots & \ldots \\
0 & 0 & 0 & 0 & 0 & \cdots & R_{u, d 1 d n}
\end{array}\right]
$$

The filter gain could be given by

$$
\mathbf{K}_{k+1}=\mathbf{P}_{k+1}^{-} \mathbf{H}_{k+1}^{T}\left[\mathbf{H}_{k+1} \mathbf{P}_{k+1}^{-} \mathbf{H}_{k+1}^{T}+\mathbf{R}_{k+1}\right]^{-1}
$$

The covariance matrix is updated by

$$
\mathbf{P}_{k+1}^{+}=\left[\mathbf{I}_{6 \times 6}-\mathbf{K}_{k+1} \mathbf{H}_{k+1}\right] \mathbf{P}_{k+1}^{-}
$$

The state is updated by

$$
\mathbf{X}_{c d 1, k+a}^{+}=\mathbf{X}_{c d 1, k+a}^{-}+\Delta \mathbf{X}_{c d 1, k+a}^{+}
$$

Where

$$
\Delta \mathbf{X}_{c d 1, k+a}^{+}=\mathbf{K}_{k+1}\left[\tilde{\mathbf{y}}_{k+1}-\hat{\mathbf{y}}_{k+1}^{-}\right]
$$

\section{Simulations}

To verify the effectiveness of the proposed estimation algorithm, simulations are conducted on a formation 
flying mission with a chief and 4 deputies. The simulation parameters are given in Table 1 , including orbit elements, relative positions, sensor accuracy, noise levels and initial relative position error.

Table 1 Simulation Parameters

\begin{tabular}{c|c}
\hline \hline Parameter & Value \\
\hline Chief 's semi-major axis, $a(\mathrm{~km})$ & $2 \times 10^{4}$ \\
Chief 's inclination, $i(\mathrm{deg})$ & 30 \\
Chief 's eccentricity, $e$ & 0 \\
Deputy 1's position relative to chief, $(x, y, z)(\mathrm{m})$ & $(300,300,500)$ \\
Deputy 2's position relative to chief, $(x, y, z)(\mathrm{m})$ & $(300,-300,500)$ \\
Deputy 3's position relative to chief, $(x, y, z)(\mathrm{m})$ & $(-300,300,500)$ \\
Deputy 4's position relative to chief, $(x, y, z)(\mathrm{m})$ & $(-300,-300,500)$ \\
Vision-sensor noise, $\phi, \psi(\mathrm{rad})$ & $2.5 \times 10^{-4}$ \\
Laser Rangefinder noise, $R(\mathrm{~m})$ & 0.5 \\
Process noise, $\mathbf{Q}$ & $1 \times 10^{-8} \mathbf{I}_{6 \times 6}$ \\
Initial relative position error, $\Delta x(\mathrm{~m})$ & 30 \\
\hline \hline
\end{tabular}

The simulation scene and formation layout are shown below:

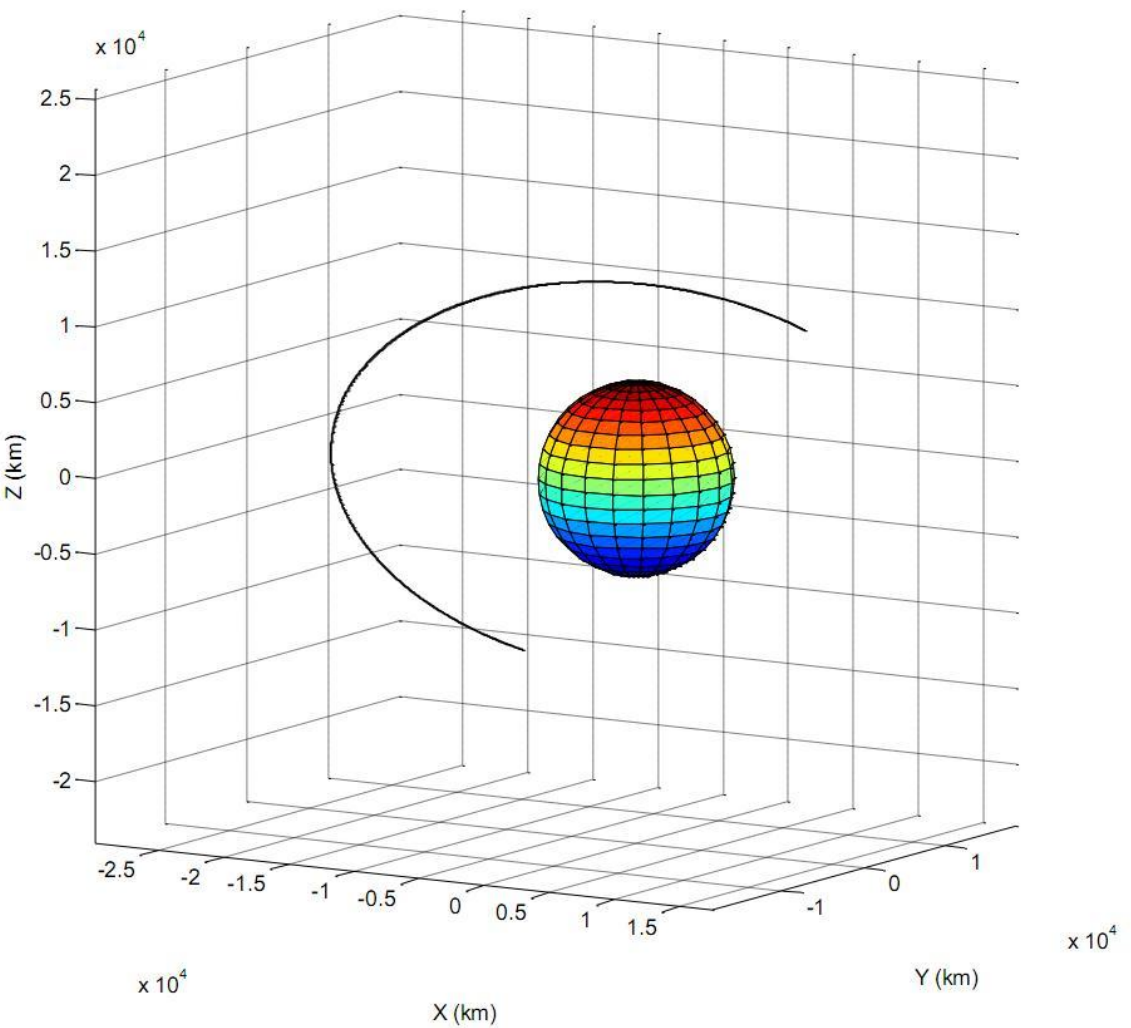

Figure 4 Simulation Scene 


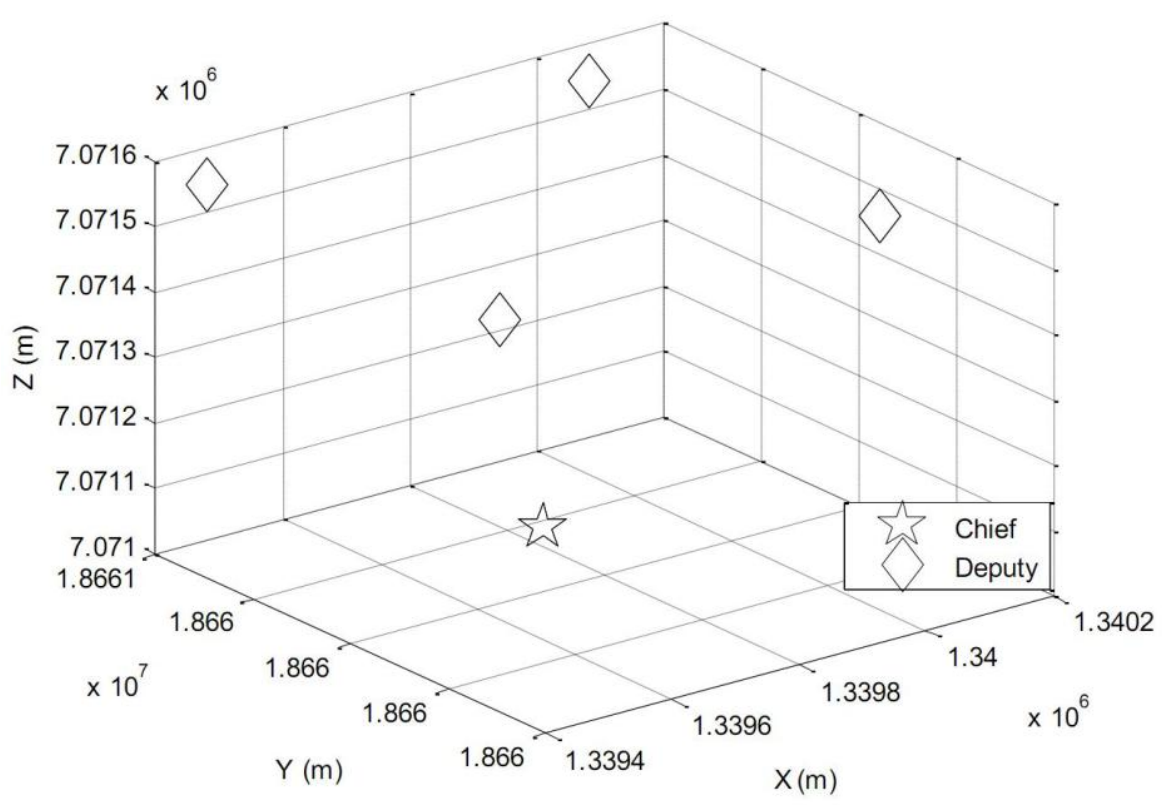

Figure 5 Formation Layout

\subsection{Simulation Result}

The following three simulation scenarios will be studied.

(a) All relative measurements are available.

(b) The line of sight between the chief and deputy 1 is unavailable.

(c) Both line of sight and range between the chief and deputy 1 are unavailable.

Monte Carlo simulation is used. Results of 50 simulations are given in Table. 2 and Figs. 6-17. From these figures, we can find that the novel decentralized relative navigation algorithm is very reliable, it can not only work in the situation when all relative measurements are available but also works in the scenario "b" and scenario "c" as well. The estimated error are small, all three axis position error are smaller than 0.1m. In Fig. 6, 10 and 14, the solid line is the relative navigation result of the deputy 1 using Line-of-sight and range between the deputy 1 and the chief. The comparison indicates that the estimation performance has been improved by fusing the ranges between the deputy 1 and other deputies which we call augmented ones. Fig. 7, 11 and 15 show the simulation results of scenario "a". It can be seen that the centralized algorithm got the best estimated result, and the novel decentralized algorithm is the second best. Furthermore, the performances of these two algorithms are very close. But the computational burden and communication burden of chief is high. Fig. 8, 12 and 14 give the simulation results of scenario "b". It can be seen that the performance of the novel decentralized algorithm is close to the iteration algorithm, 
but the estimation converges faster than non-iteration algorithm. The reason is that the proposed novel algorithm accounts for the uncertainty in other spacecraft states. However, the novel decentralized algorithm needs not to compute iteratively. Similar situation happens in Fig. 9, 13 and 17 which show the simulation results of scenario "c". The relative navigation errors of X-axis position of the deputy 1 using different filtering are shown in Tab. 2.

\section{Conclusions}

In this paper, a novel decentralized estimation algorithm is developed for spacecraft formation flying. The relative measurements contain not only the line of sight and range between the deputy and the chief, but also the ranges between the deputies. Therefore, the states of other spacecraft are required in the decentralized filter. To account for the uncertainty in the estimated states of other spacecraft, a sigmapoint method is applied in the filter design. The simulations were conducted and the results showed that by accounting for the uncertainty in the states of other spacecraft using sigma-point method, the proposed novel decentralized algorithm dose not compute iteratively, thus uses less computational cost, but almost the same accuracy which compared to the iterated algorithm.

\section{References}

[1] P. Sengupta, S. R. Vadali, and K. T. Alfriend. Averaged relativemotion and applications to formation flight near perturbed orbits. Journal of Guidance, Control and Dynamics, 31(2):258-272, 2008.

Table 2 Relative Navigation Accuracy using Different Filter Methods

\begin{tabular}{c|c|c|c}
\hline \hline Filter Method & Condition 1 & Condition 2 & Condition 3 \\
\hline Centralized filtering & 0.0076 & 0.0487 & 0.0621 \\
Novel filtering & 0.0086 & 0.0633 & 0.0941 \\
Iteration (4 times) & 0.0240 & 0.0642 & 0.0952 \\
Iteration (0 times) & 0.0270 & 0.1012 & 0.1307 \\
\hline \hline
\end{tabular}




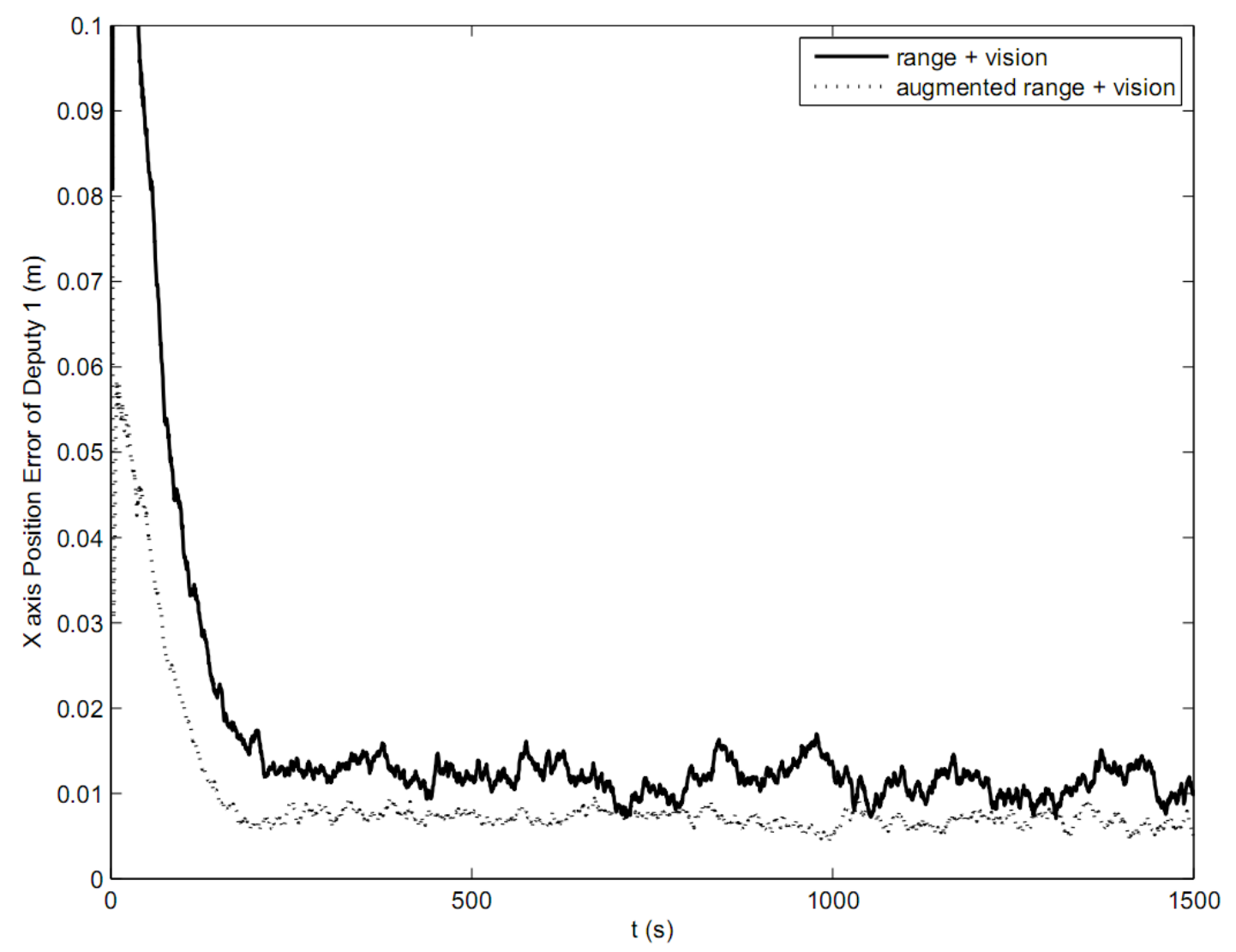

Fig. 6 Comparison of Relative Navigation Errors with/without Augmented Ranges

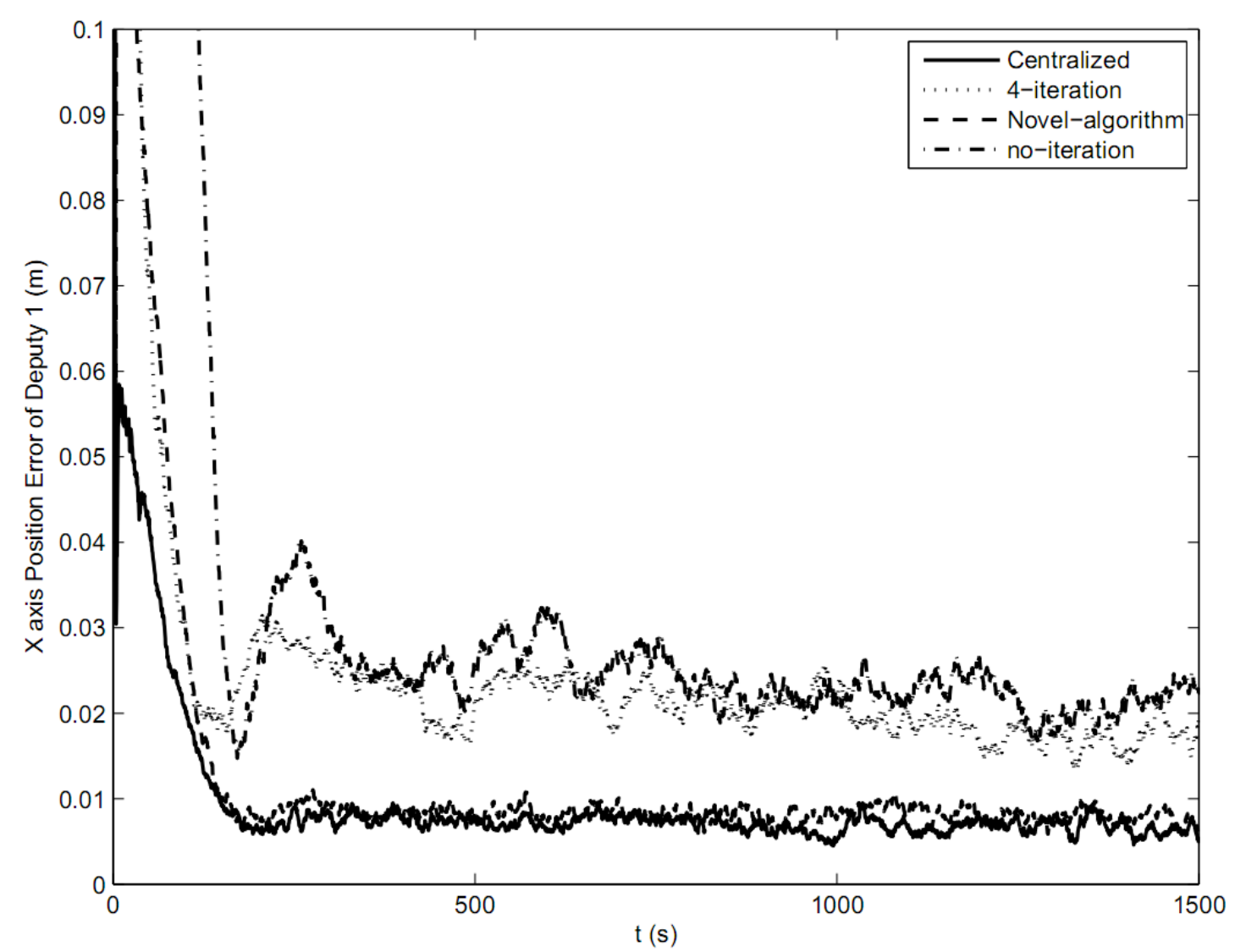

Fig. 7 Comparison of Relative Navigation Errors in scenario (a) using Different Algorithms 


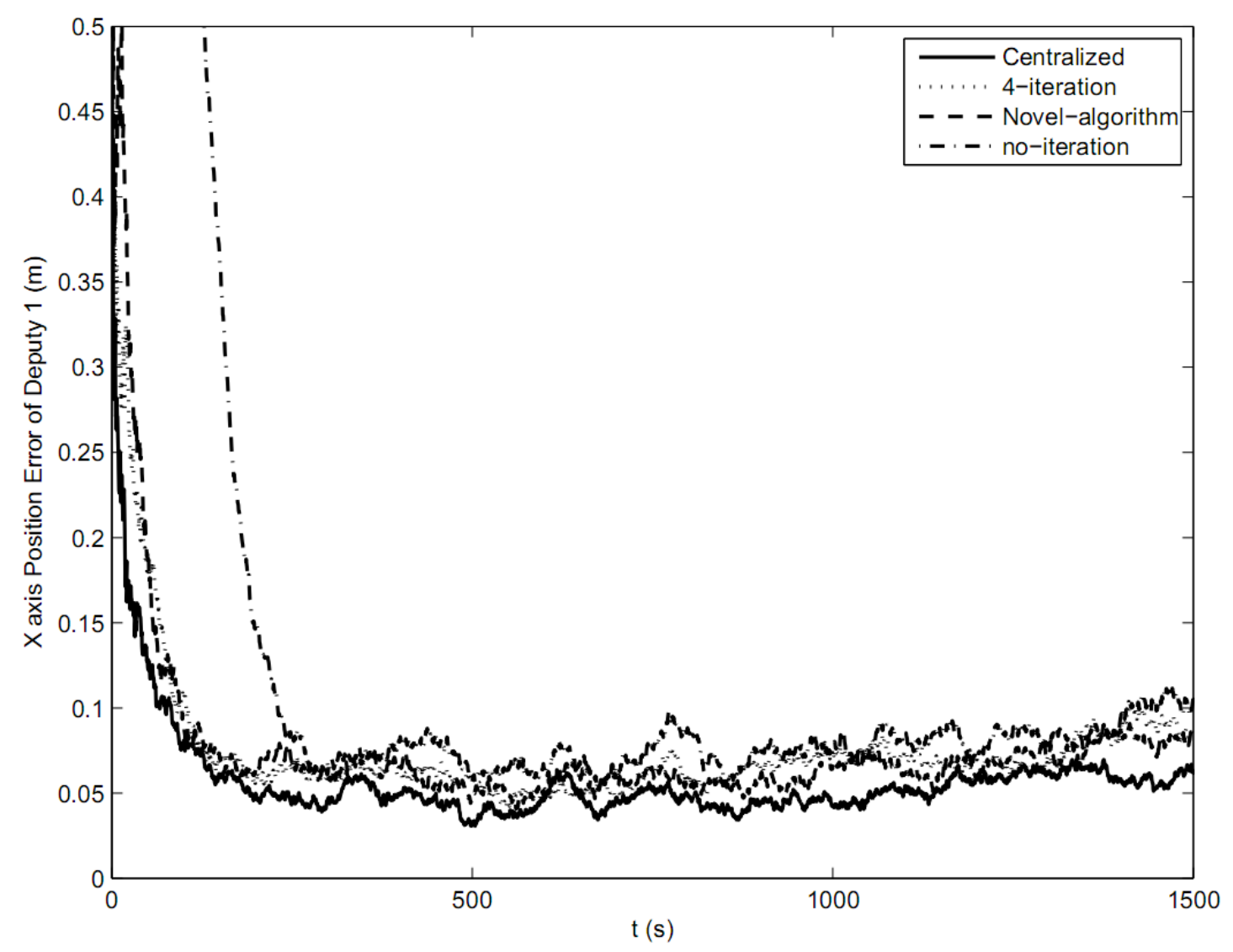

Fig. 8 Comparison of Relative Navigation Errors in scenario (b) using Different Algorithms

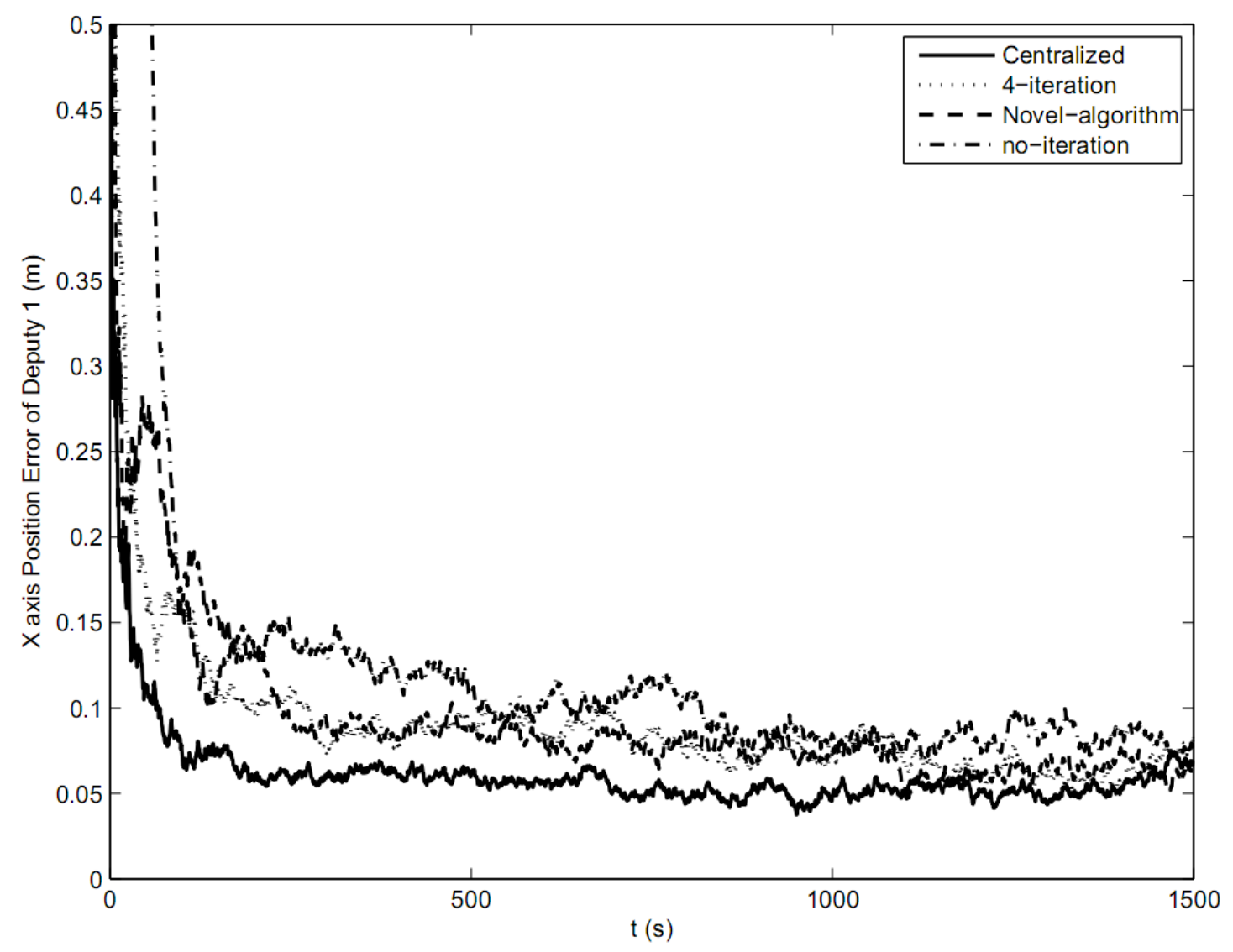

Fig. 9 Comparison of Relative Navigation Errors in scenario (c) using Different Algorithms 


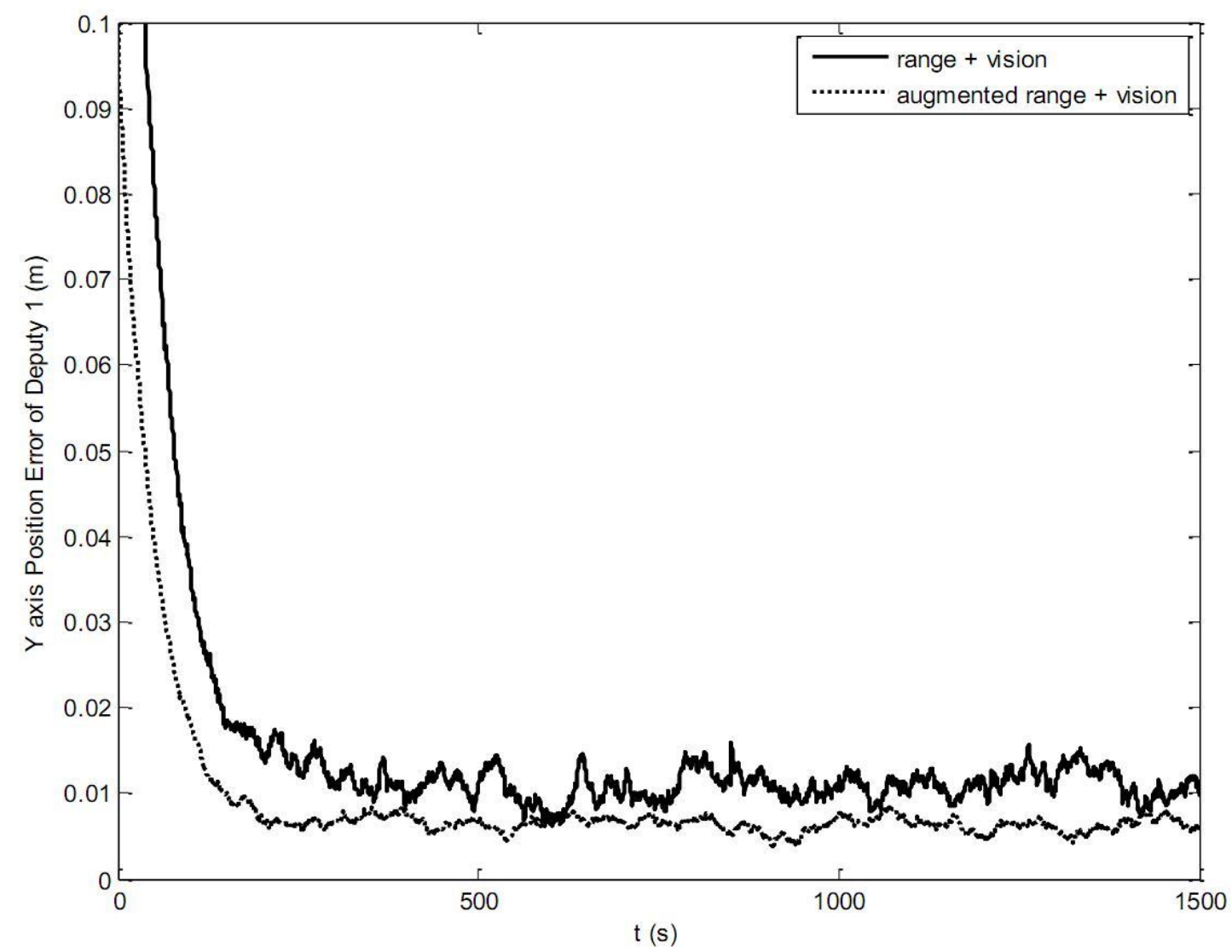

Figure 10 Comparison of Relative Navigation Errors with/without Augmented Ranges

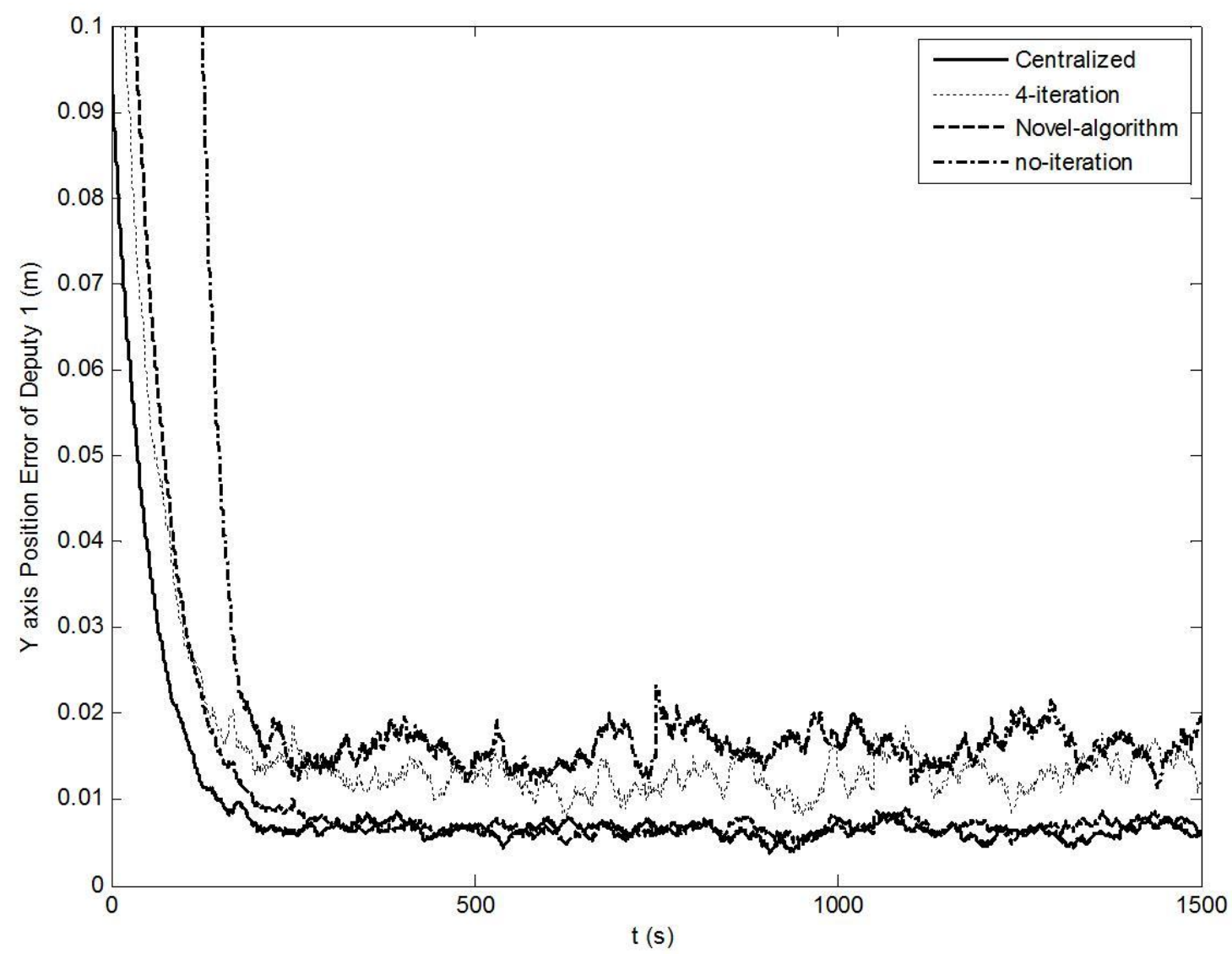

Figure 11 Comparison of Relative Navigation Errors in scenario (a) using Different Algorithms 


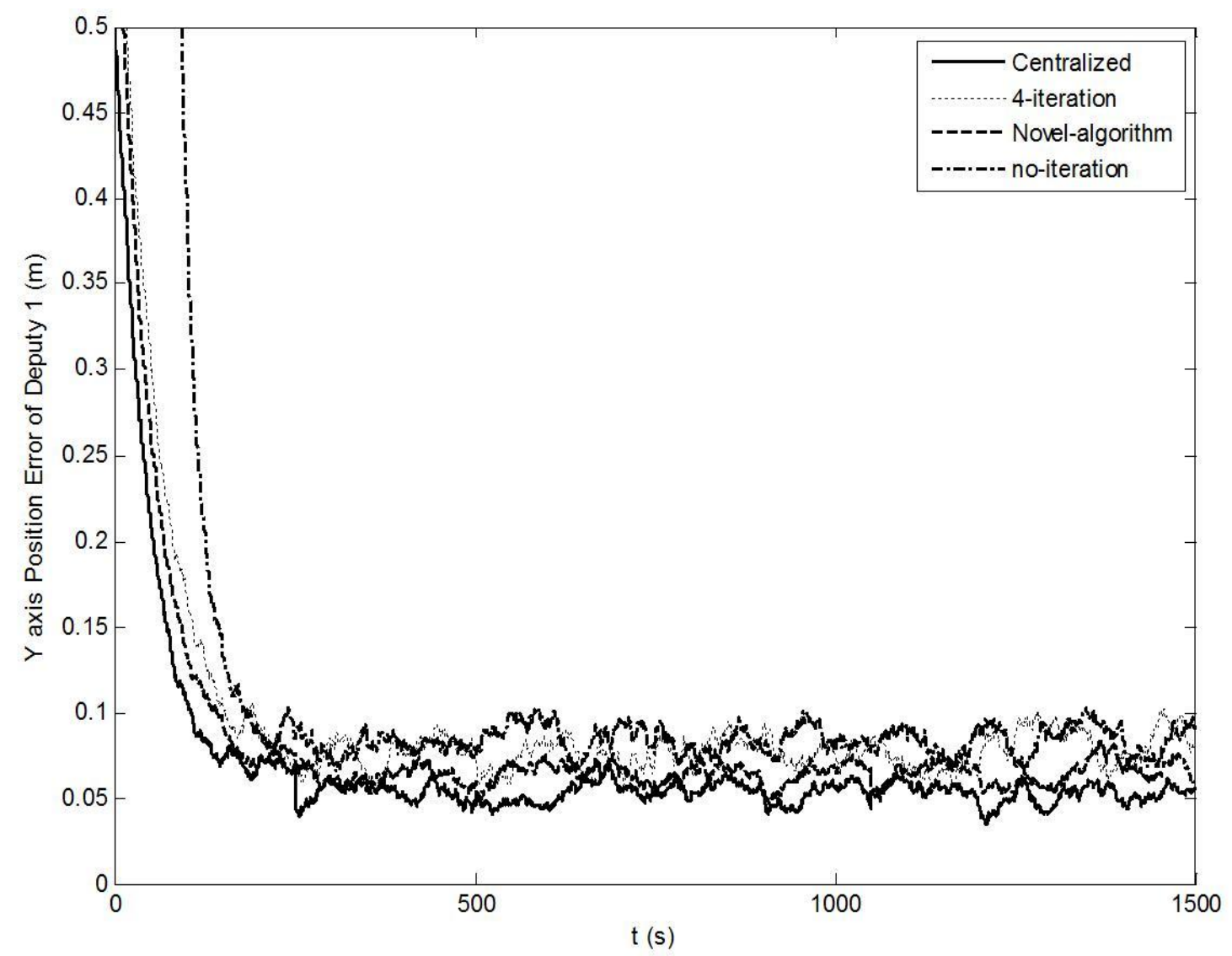

Figure 12 Comparison of Relative Navigation Errors in scenario (b) using Different Algorithms

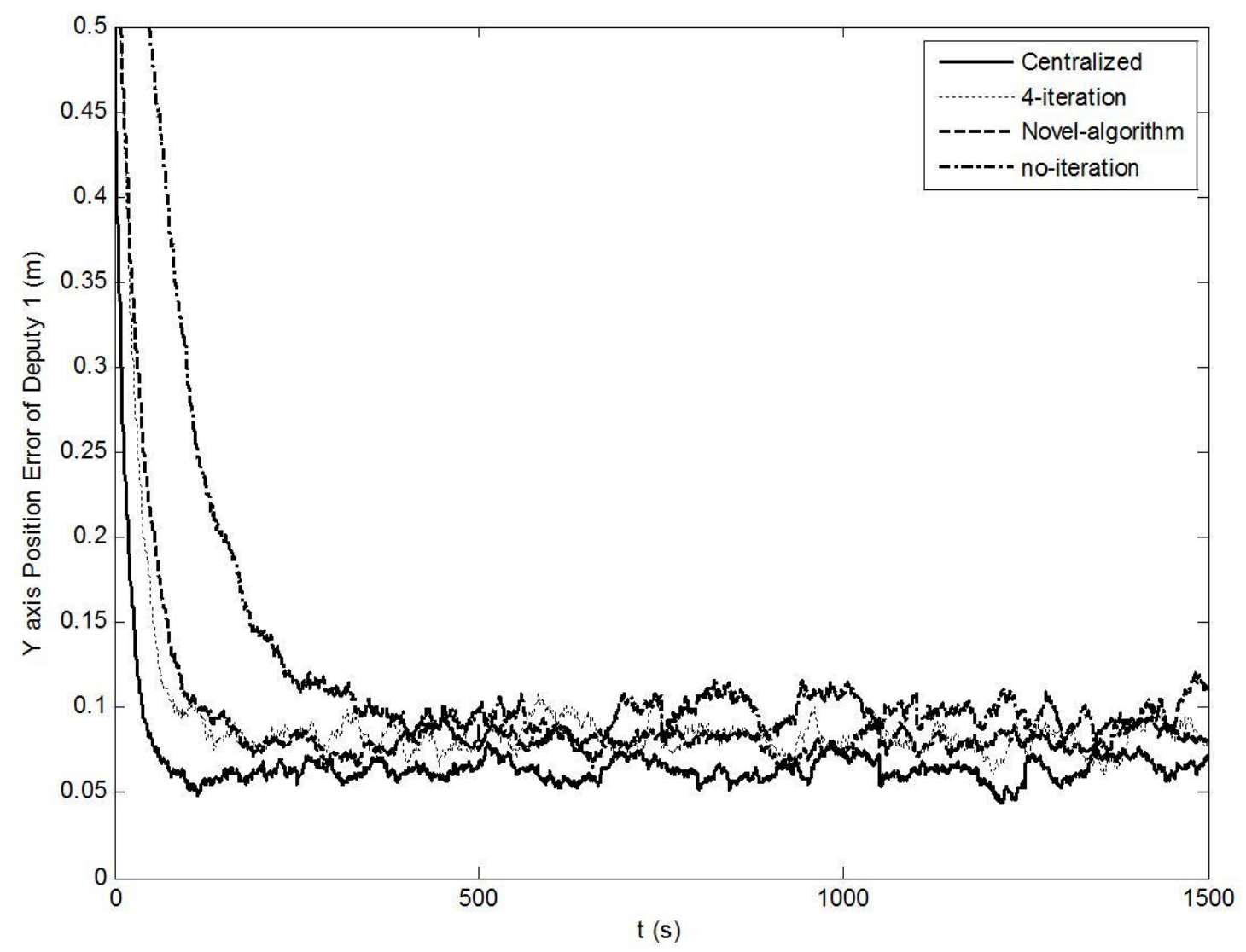

Figure 13 Comparison of Relative Navigation Errors in scenario (c) using Different Algorithms 


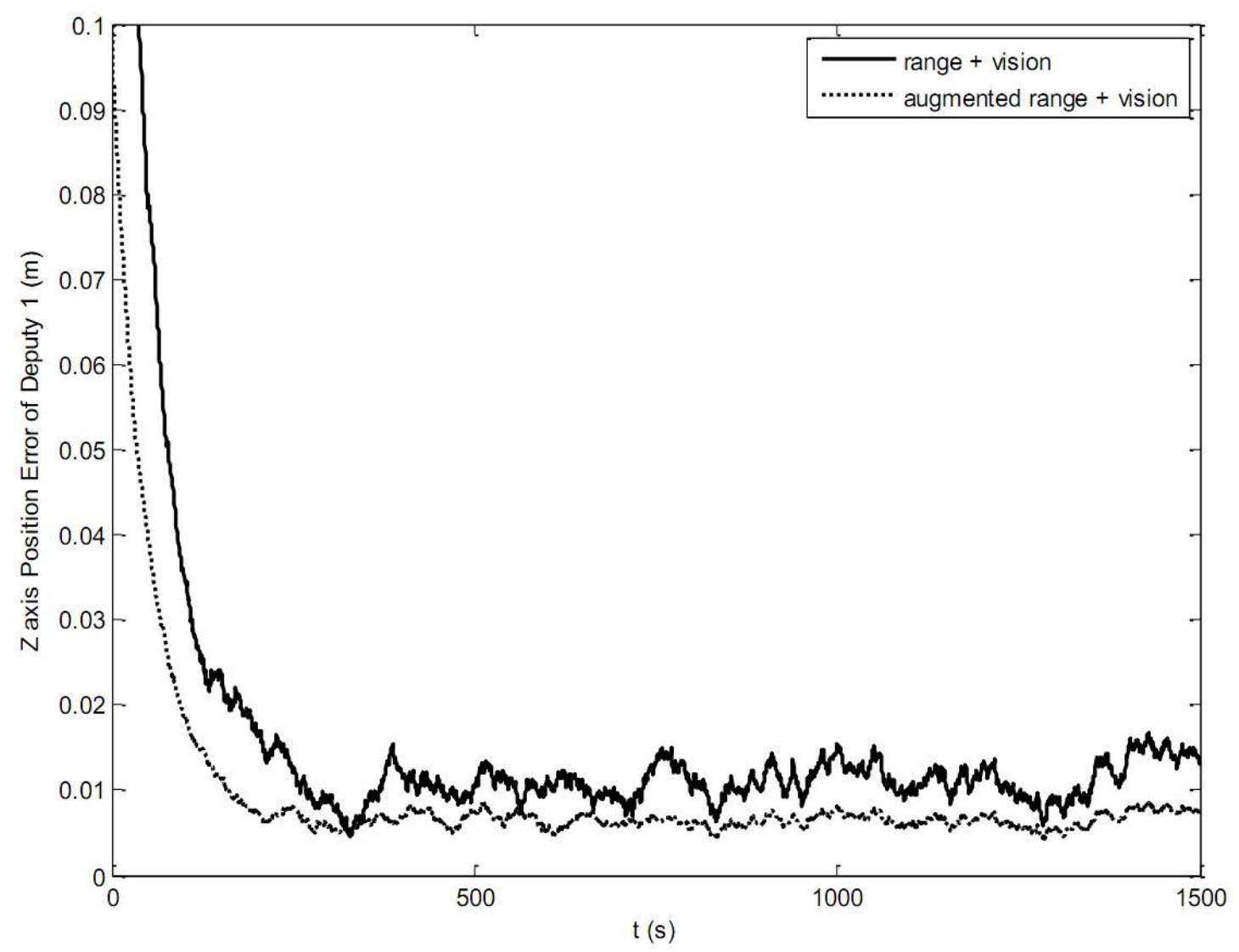

Figure 14 Comparison of Relative Navigation Errors with/without Augmented Ranges

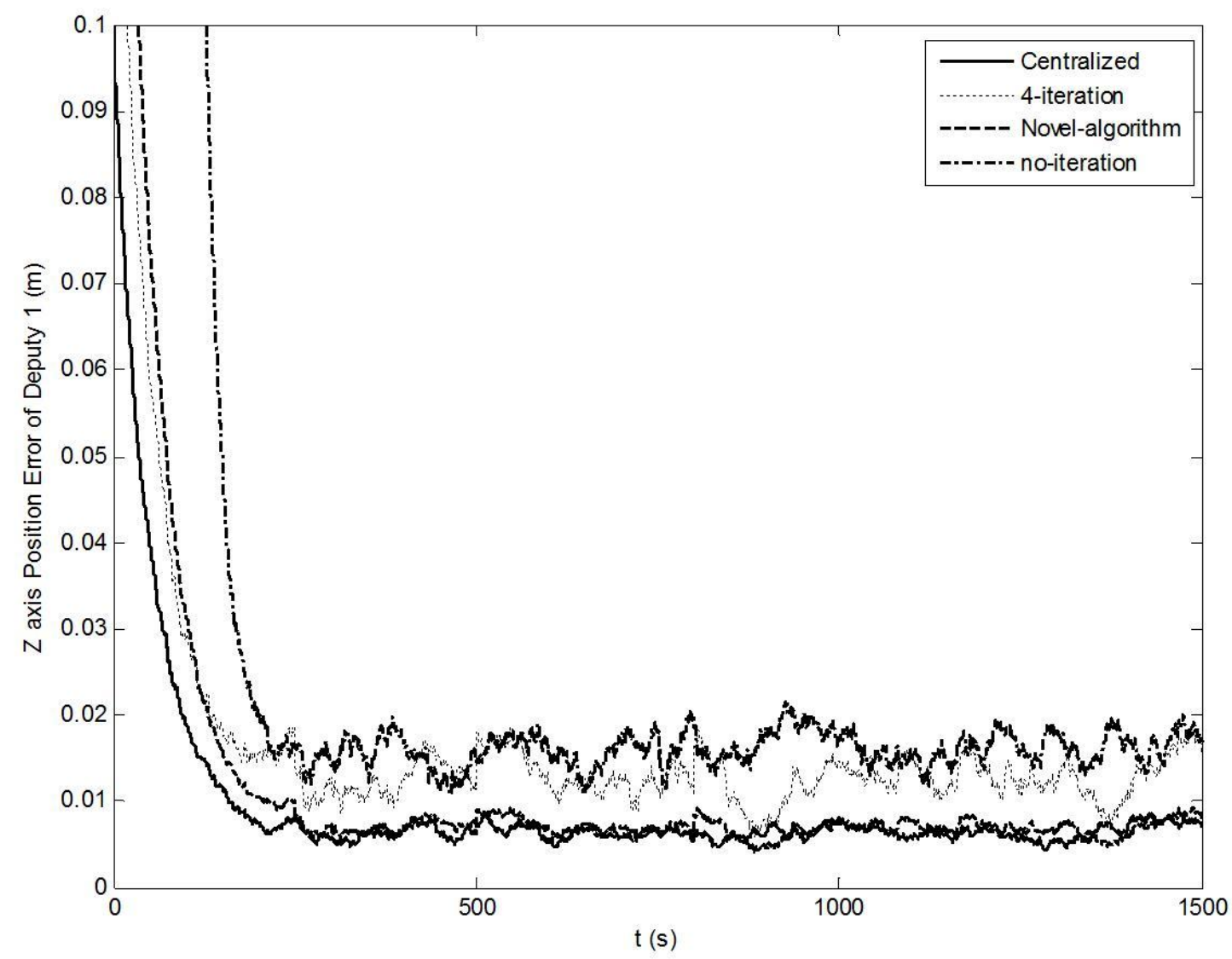

Figure 15 Comparison of Relative Navigation Errors in scenario (a) using Different Algorithms 


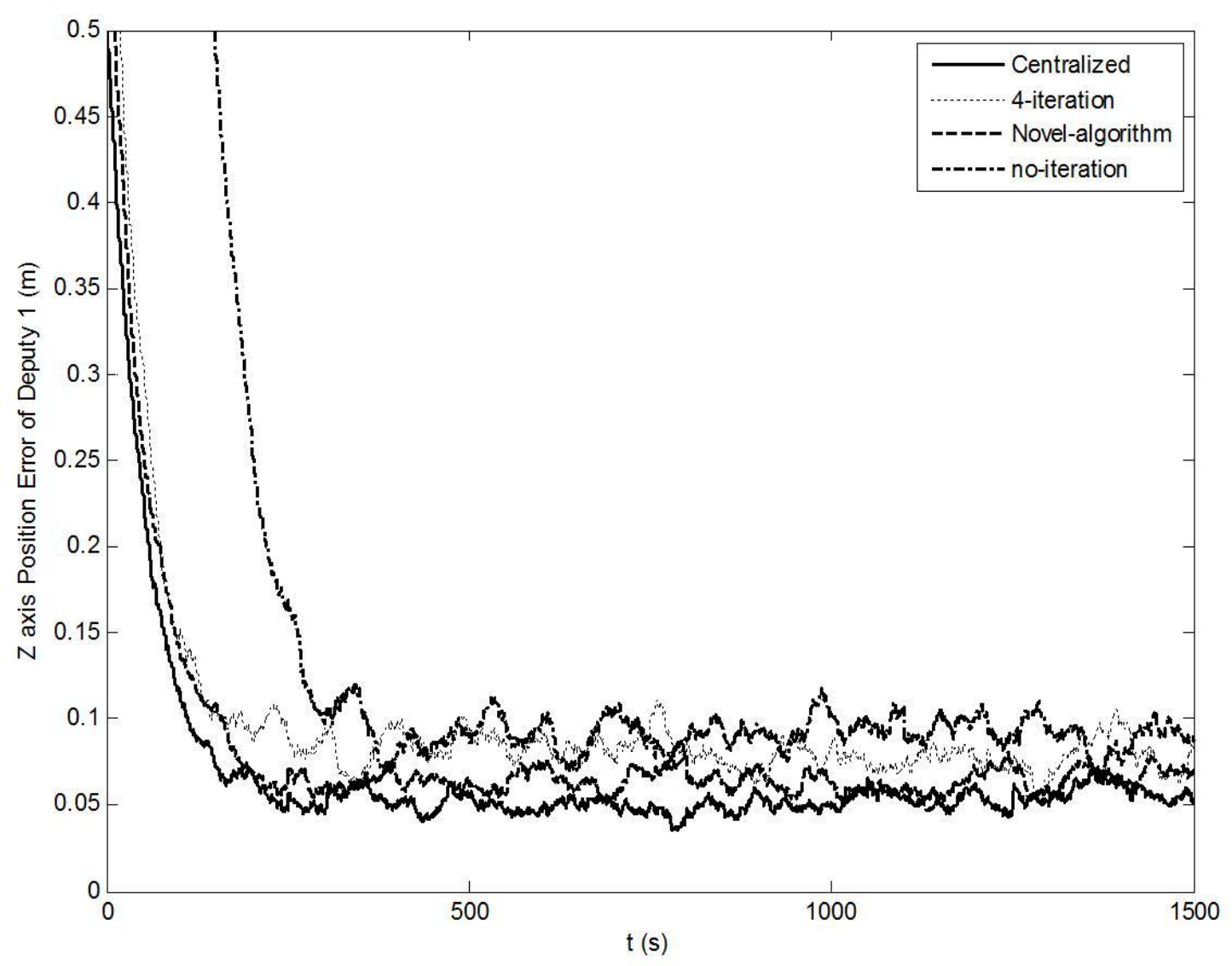

Figure 16 Comparison of Relative Navigation Errors in scenario (b) using Different Algorithms

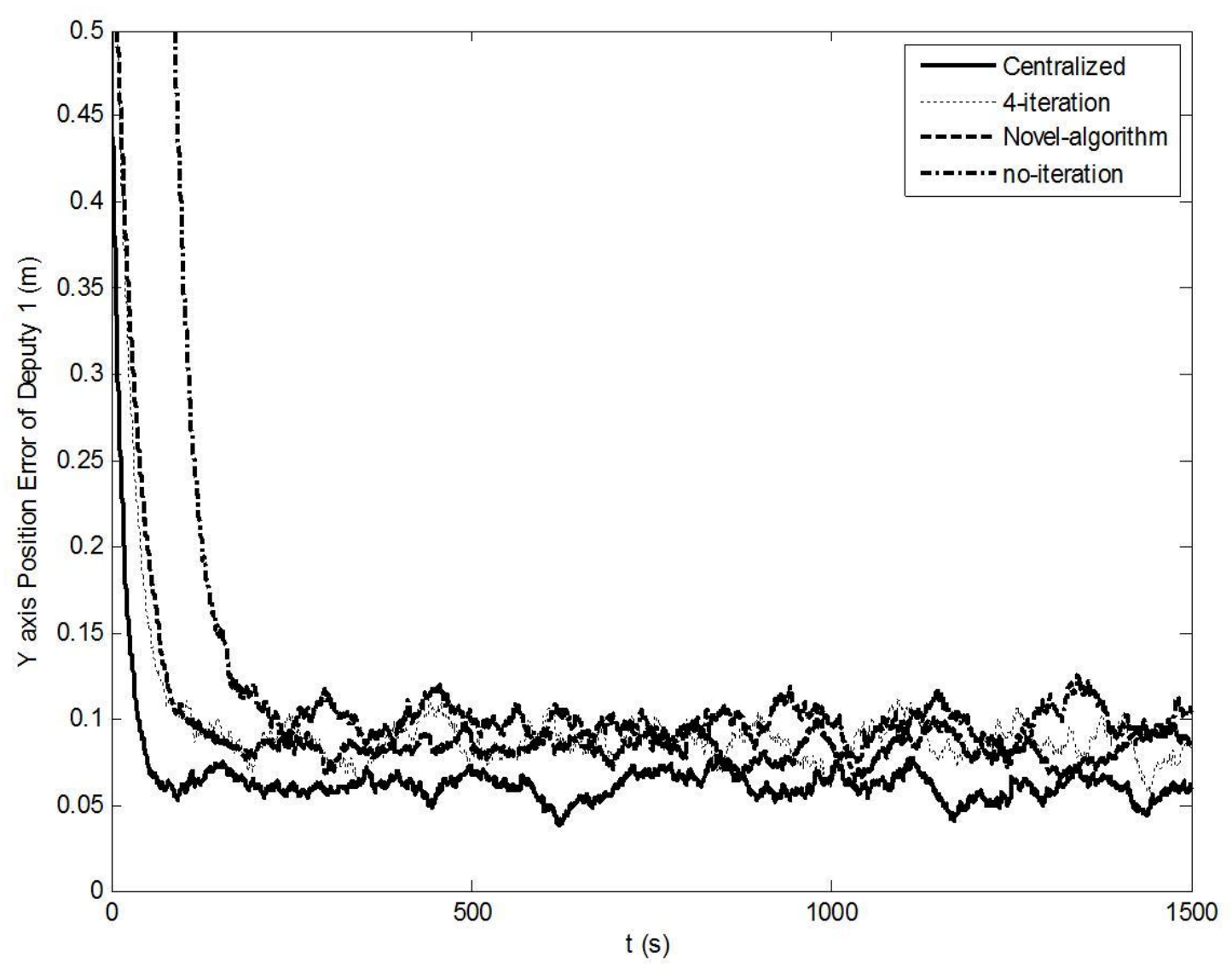

Figure 17 Comparison of Relative Navigation Errors in scenario (c) using Different Algorithms 
[2] G. Inalhan, M. Tillerson, and J. P. How. Relative dynamics and control of spacecraft formations in eccentric orbits. Journal of Guidance, Control and Dynamics, 25(1):48-59, 2002.

[3] J. L. Garrison and P. Axelrad. Relative navigation in elliptical orbits using an iteration nonlinear filter. In Proc of the ION GPS-97 Conference, Kansas, MO, Sept. 1997.

[4] G. Zhang, and M. Kontitsis et al. Cooperative Relative Navigation for Space Rendezvous and Proximity Operations using Controlled Active Vision. Journal of Field Robotics, Mar 2015, pp.1-24.

[5] D. B. Wilson, A. H. Goktogan, and S. Sukkarieh. A Vision Based Relative Navigation Framework for Formation Flight. In 2014 IEEE International Conference on Robotics \& Automation (ICRA), HK, China, June. 2014.

[6] R. C. Leishman, T. W. McLain and R. W. Beard. Relative Navigation Approach for Vision-Based Aerial GPS-Denied Navigation. Journal of Intelligent \& Robotic Systems, 74(2014), pp. 97-111.

[7] O. Montenbruck, T. Ebinuma, E. G .Lightsey, and S. Leung. A real-time kinematic gps sensor for spacecraft relative navigation. Aerospace Science and Technology, 6(6):435-449, 2002.

[8] T. Corazzini, A. Robertson, J. C. Adams, A. Hassibi, and J. P. How. Gps sensing for spacecraft formation flying. In Proc of the ION GPS-97 Conference, Kansas, MO, Sept. 1997.

[9] G. Purcell, D. Kuang, and S. Lichten etc. Autonomous formation flyer (aff) sensor technology development. In ASS Guidance and Control Conference, Breckenridge, CO, Feb. 1998. AAS-98-062.

[10] S. G. Kim, J. L. Crassidis, Y. Cheng, and A. M. Fosbury. Kalman filtering for relative spacecraft attitude and position estimation. Journal of Guidance, Control and Dynamics, 30(1):133-145, 2007.

[11] R. Alonso, J. Y. Du, D. Hughes, J. L. Junkins, and J. L. Crassidis. Relative navigation for formation flying of spacecraft. In Proceedings of the Flight Mechanics Symposium, Greenbelt, MD, Jun. 2001. NASA-Goddard Space Flight Center.

[12] P. Ferguson and J. P. How. Decentralized estimation algorithms for formation flying spacecraft. In AIAA Guidance, Navigation, and Control Conference and Exhibit, Austin, TX, Aug. 2003. 
[13] C. W. Park. Precise Relative Navigation using Augmented CDGPS. PhD thesis, Stanford University, Dept. of Mechanical Eng, Jun. 2001.

[14] F. D. Busse. Precise Formation-State Estimation in Low Earth Orbit using Carrier Differential GPS. $\mathrm{PhD}$ thesis, Stanford Universiy, Dept. of Aeronautics and Astronautics, Nov. 2002.

[15] P Z. Zhang, C. Lin and B. Chen. New Decentralized $\mathrm{H} \infty$ Filter Design for Nonlinear Interconnected Systems Based on Takagi-Sugeno Fuzzy Models. IEEE Transactions on Cybernetics, 99(2015), pp.1-11.

[16] A. Mohammadi, and K. N. Plataniotis. Structure-Induced Complex Kalman Filter for Decentralized Sequential Bayesian Estimation. IEEE Signal Processing Letters, 22(2015), pp. 1419-1423.

[17] X. Qing, and H. R. Karimi et al. Decentralized unscented Kalman filter based on a consensus algorithm for multi-area dynamic state estimation in power systems. International Journal of Electrical Power \& Energy Systems, 65(2015), pp. 26-33.

[18] S. Julier and J. Uhlmann, Unscented filtering and nonlinear estimation. In Proceedings of the IEEE, 92(3), pp. 646-655 (March 2004).

[19] R. V. D. Merwe. Sigma-Point Kalman Filters for Probabilistic Inference in Dynamic State-Space Models. PhD thesis, Oregon Health Science University, the OGI School of Science Engineering, Apr. 2004.

\section{Acknowledgments}

This work has been sponsored by project 61304236 supported by National Science Foundation of China. 